RESEARCH REPORT

\title{
A CROSS-SECTIONAL SURVEY BASED ON PERCEPTION OF CAREGIVERS TO COMMUNICATION BARRIERS WITH AUTISM SPECTRUM DISORDER CHILDREN
}

\begin{abstract}
BACKGROUND AND AIMS

Communication has been a hallmark in autistic children with verbal and non-verbal language difficulties. These challenges may lead towards the development of syntactic structure that may cause understanding issues and communication breakdown. Therefore, the purpose of this study is to determine the communicative barriers in ASD children and their parents.
\end{abstract}

\section{METHODOLOGY}

This cross-sectional survey was conducted at the special education schools of Karachi; enrolled 56 parents of ASD children using non-probability convenience sampling technique. Data was collected through 'communicative difficulties questionnaire.

\section{RESULTS}

It was reported that $40.5 \%$ parents have difficulties with their child while $57 \%$ to $59.5 \%$ were found to be upset with communication difficulties. $24.1 \%$ parents have impression that their child doesn't understand what they say.

\section{CONCLUSION}

Varied percentage was observed in communicative domains of the questionnaire that showed high proportion of communication barriers between parents and child. Further studies are needed to analyze the communication between parents and autistic children to address its impact.

\section{KEY WORDS}

Autism Spectrum Disorder, Fragile X-Syndrome, Motivation Behavior, Caregivers, Perception

\author{
Anum Zaid \\ Physiotherapist \\ Pakistan Centre of Autism \\ anamsyed2@gmail.com
}

[Zaid A. Cross-sectional Survey based on Perception of Caregivers to Communication Barriers with Autism Spectrum Disorder Children. Pak. j. rehabil.2019;8(2):26-31] 


\section{INTRODUCTION}

Autism Spectrum Disorder, referred to as ASD is a neurological and developmental disability that has been increasing worldwide from the past three decades i.e. approximately 1 out of 55 children has been diagnosed with the disorder annually? According to Developmental Disabilities Monitoring Network (ADDM), the current global prevalence of autism has been identified as $2.3 \%$ whereas an average prevalence of autistic children is $1-2 \%$ in developing countries ${ }^{2}$. A number of studies conclude that among all the disabilities, autism is characterized as an intellectual disability that affect the communication and social interaction which may lead to behavior challenges ${ }^{1-3}$. Furthermore, it was advocated that the most dominant problems in ASD individuals are functional communication and social association followed by difficulty in mainstream pattern of routine work and restricted interests ${ }^{4}$. In addition, these difficulties in verbal and non-verbal communication are developed due to genetic inheritance of ASD by their ancestors that may results in complex risks factors ${ }^{5}$. Moreover, stratification of genetic factors in Gastrointestinal Disorders among autistic children has demonstrated a similar pattern that showed association with communication and social phenotypes ${ }^{6}$. It was also indicated that at least two to three traits of affected domains may lead to poor sensory integration and communicative barriers ${ }^{7}$. A study was conducted considering the autistic children pre-natal factors revealed that maternal gestational age, number of births and insults may have strong association with developmental disabilities thereby leads to a multifunctional disease ${ }^{6-8}$. Individuals with communication disorder deals with the lack of speech in children younger than 3 years present with unusual feature like echolalia, contrary lack of facial expression to the straight away and absence of speech?. Despite, parents anti-social behavior and lack of communication skills results the dependency for their child's basic needs resulting in lifelong concern for the both children and parents ${ }^{10}$. The continuous stress of parents having autistic children is higher than those parents who have children with other disabilities like Down syndrome, behavior disorder and fragile $X$-syndrome etc ${ }^{11}$. On the other hand, depressed parents may affect child's treatment effectiveness of early intervention due to lack of communication ${ }^{9-11}$. Yet, most of the times parent do not understand the demand or need of child. One more underlying issue concerning ASD is the parent's perception about the acceptance having an autistic child by the society ${ }^{12}$. Literature showed that accusation may end up with depression, lack of self-esteem and separation from the society ${ }^{13}$.

Communication has been a hallmark in autistic children with verbal and non-verbal language difficulties ${ }^{14}$. These challenges may lead towards the development of syntactic and lexical structure that may cause understanding issues and communication breakdown ${ }^{15}$. Multiple studies revealed that most of the ASD children, in particular with the issues of non-verbal communication have shorter engagement, restricted gestures as well as difficulties in consonant production ${ }^{16}$. Although, communication in autistic children can be evaluated through variety of methods that may include formal and informal assessments using standardized tests or questionnaires, parent-children interviews, and functional language evaluation ${ }^{17}$ although, poor intent in communication and difficulty in practicability of assessment leads to unreliable results in ASD children. However, among variety of assessments, speech analysis has provided better results of functional communication in ASD children ${ }^{17}$. In clinical practice, communication between caregiver or ASD children is evaluated based on the child performance by a test or parental-report ${ }^{18}$. In context, this practice is convenient to administer and time effective as well. Despite of the fact, the precision and reliability of parental responses is ambiguous. Number of researches indicated that the communication is a challenge to assess in an unfamiliar environment due to inadequate motivation to communicate and inadequate verbal output ${ }^{14-18}$. In addition, evaluation of pragmatics is relatively more challenging among non-verbal children involved in a restricted environment. In this regard, Salcuni et $\mathrm{al}^{19}$ suggested that in order to gather adequate and representative sample for profiling communication of ASD children, it is evident to consider children behavior during an activity, particularly with a familiar partner that may increases the scope to promote positive behavior in collaboration such as engagement in during playing, sharing or laughing. Studies also reported a strong association between functional activity and language pragmatics that is crucial to enhance cognition in autism that may lead to better understanding of linguistics ${ }^{20}$. Therefore, playing activities of 25-30 minutes is recommended to provide a better insight of child communication profile and skills for more reliable finding ${ }^{21}$. The communication gestures that include speech and voice intonations are influenced by the caregivers at large due to culture-developmental context. Previous studies have explored that parental attitudes and parenting aspects typically influenced the child's communicative behavior ${ }^{18}$. Such a spectrum of language pragmatics with significant communicative limitation makes it more challenging to gather a representative sample for diagnostic and intervention purposes in ASD population ${ }^{18-20}$. However due to scarcity of studies, influence of environment, peers and caregivers on ASD children communication profile is still unknown. Therefore, the purpose of this study is to determine the communication barriers in ASD children and their parents to evaluate their interaction, needs and demands in order to facilitate the children in rehabilitation. 


\section{METHODOLOGY}

This cross-sectional survey was conducted at the special education schools of Karachi. A total sample of 56 parents or caregivers of ASD children were enrolled using non-probability convenience sampling technique in the tenure of 6 months based on the following criteria:

\section{INCLUSION \& EXCLUSION CRITERIA}

Primary caregivers such as parents or guardians of ASD children enrolled in rehabilitation for $>1$ year will be included where as parents of those children who are not enrolled in rehabilitation program will be excluded.

\section{DATA COLLECTION TOOL 22}

Data was collected through the questionnaire about communicative difficulties between parents and ASD children. The questionnaire is a likert type scale comprises of 24 questions that are divided in 4 domains, 1stand 3rddomain represents the personal level and personality whereas 2ndand 4thdomain represents the perception of parents/guardians regarding interaction between their children and society. Further the responses of the questions will be answered in four categories consisted of "completely agree", "agree", "completely disagree" and "disagree".

\section{DATA COLLECTION PROCEDURE}

Participants were given consent forms prior to study for voluntarily participation. The information regarding questionnaire and survey was delivered to ensure their understanding whereas it was ascertained that participants' identity or shared material was kept confidential and shall not be disclosed or used without permission.

\section{DATA ANALYSIS}

Data entry and analysis was conducted on SPSS (Statistical Package for Social Sciences) version 20. Furthermore, descriptive statistics consists of MeantS.D. will be used to demonstrate participant's characteristics whereas participant's responses on questionnaire will be represented as frequencies and percentages.

\section{RESULTS}

A total number of 56 participants consist of 20 males and 36 females with mean age of $41.1 \pm 7.5$ years enrolled in the study. The responses of parents of children with ASD were taken on the 4 domains of questionnaire.

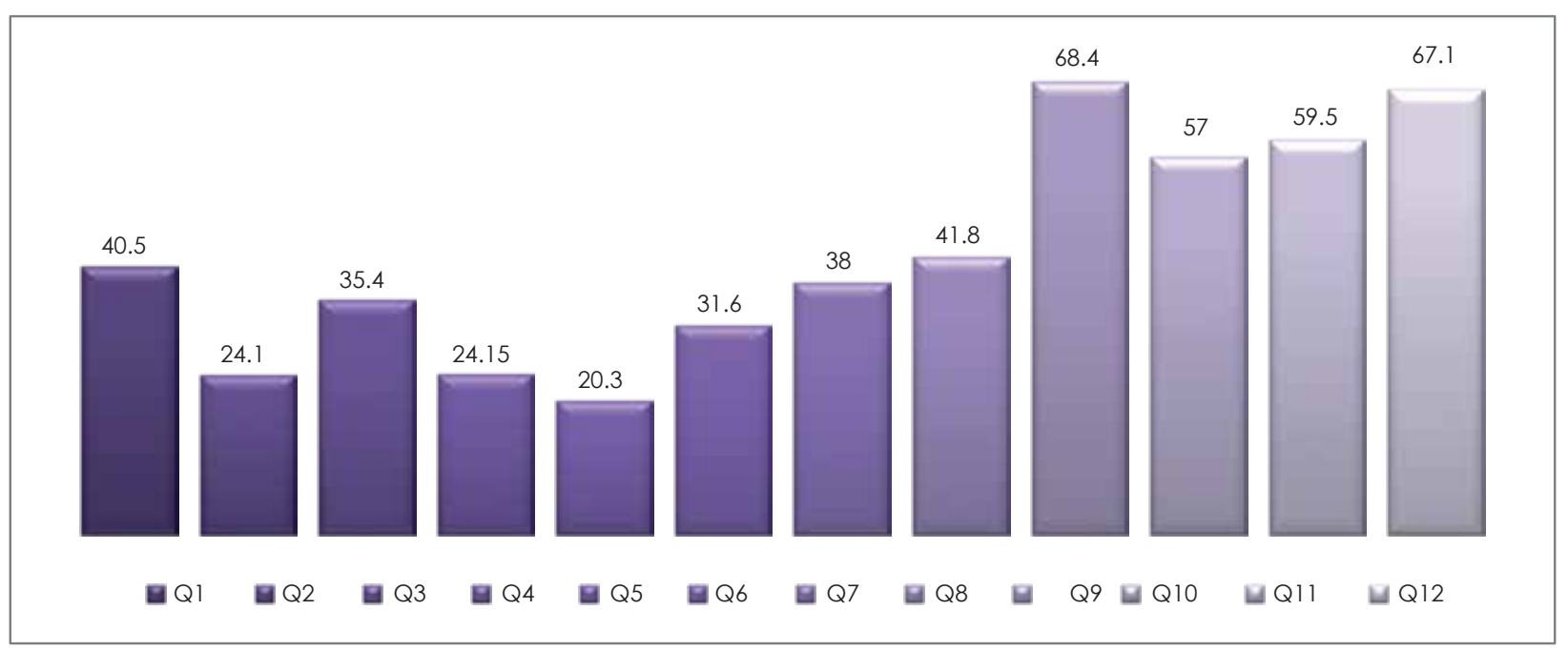

Figure-1 represents parent's/caregiver's impression about them regarding their child

Q1 I have difficulties communicating with my son/daughter

Q2 I have difficulties communicating with my son/daughter when there are just the two of us

Q3 I have difficulties communicating with my son/daughter when there are other persons in the same room

Q4 I have difficulties playing with my son/daughter

Q5 I have difficulties to understand what my son/daughter wants

Q6I have difficulties to understand what my son/daughter feels

Q7 I don't know what to do when my son/daughter doesn't understand me or when I don't understand him/her

Q8 I am not at ease with my son/daughter in public places

Q9 I worry about my son's/daughter's future

Q10 I get upset when I notice my son/daughter doesn't initiate communication

Q11 I get upset with my son's/daughter's apathy or agitation

Q12 I would like to receive more information about how to communicate with my child 
The 2nd domain of questionnaire comprised of 4 questions regarding parent's perception about people acceptance of their child stated that $59.5 \%$ parents do not understands what their child want to express. However, $40.5 \%$ parents have the impression that people avoid their child. It was also reported that $53.2 \%$ parents noticed that people think their child is strange as shown in Figure-2.

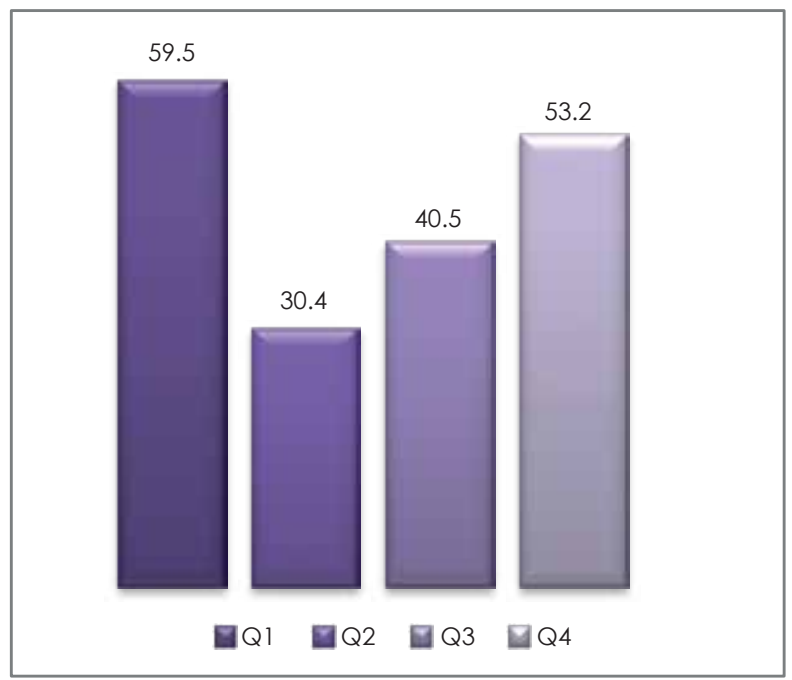

Figure 2. Represents parents' perception about other people's acceptance of their child

Q1 I feel that other people do not understand what my son/daughter wants do express

Q2 I notice that some people make fun of my son/daughter when he/she tries to express something

Q3 I have the impression that people avoid my son/daughter

Q4 I notice that people think my son/daughter is strange

Similarly, 3rd Domain included 4 questions about parents' attitude regarding their child reported that 48.1\% parents don't know how to act with their child's behavior as well as $64.6 \%$ has difficulty to interpret their child needs whereas $20.3 \%$ are unable to taught new things to their child as depicted in Figure-3.

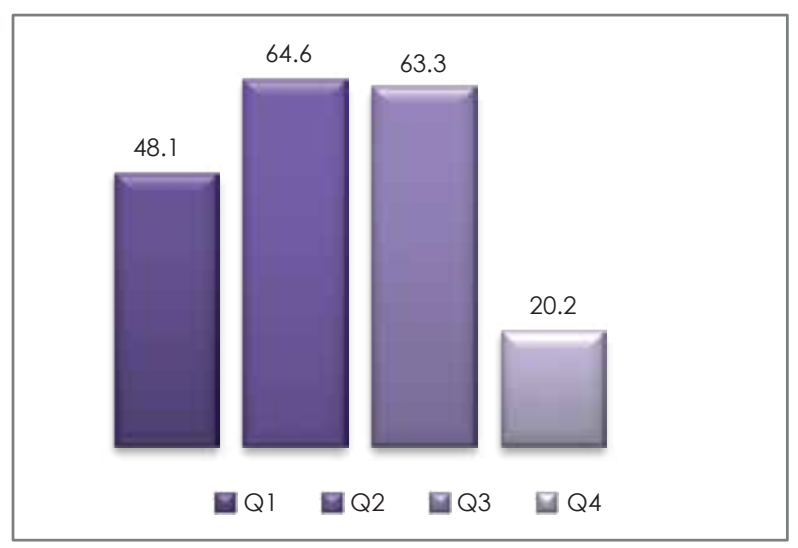

Figure 3. Represents parents' attitude regarding their child

Q1 I don't know how to act with some of my child's behavior

Q2 I reach the objects that my son/daughter points to Q3 I always talk to my son/daughter even if he/she doesn't talk to me

Q4 I can't teach new things to my son/daughte

The last domain of communicative difficulties, parent's impression about their child was reported. It was observed that $24.1 \%$ parents have impression that their child did not understand what they say. Further, $41.8 \%$ parents noticed that their child speak things that are not adequately to the moment while $51.9 \%$ parents reported that their child has few friends. The details are represented below in Figure-4.

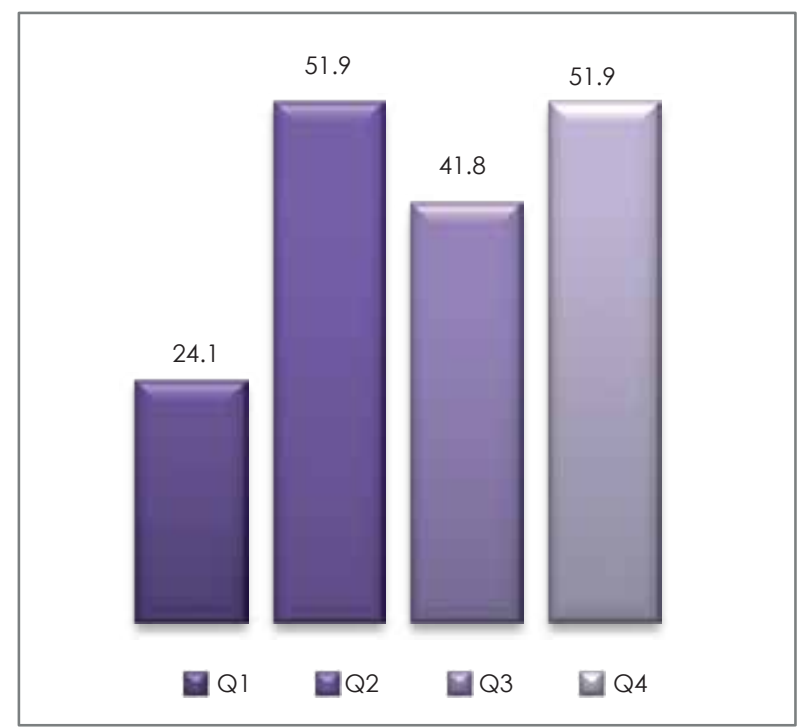

Figure 4. Parents impressions about their child

Q1 I have the impression that my son/daughter doesn't understand what I say

Q2 I have the impression that my son/daughter doesn't understand what other people say

Q3 I notice that my son/daughter speaks things that are not adequate to the moment or the context Q4 I have the impression that my son/daughter has few friends

\section{DISCUSSION}

This cross-sectional study was conducted to determine the communicative disorders between parents and children of ASD. Upon evaluation, it was revealed that varying percentage of difficulties exists between communication of children with parents or vice versa. It was concluded that a language barrier was one of the most potent finding in communicative disorders among ASD children that showed certain verbal and non-verbal difficulties. Several studies indicated that there are certain underlying environmental and genetic factors that are markers for the language barrier assumption in ASD children ${ }^{18-20}$. It was advocated that language pragmatics is poorly developed in 
children. This may be due to neurophysiological abnormalities in the brain structures, although the pathogenesis is still unknown²2.

Literature stated that several differences in language development and communication are the hallmarks in ASD children ${ }^{18-20}$. These differences may occur due to absence or delay in the language, conversational deficits, repetitive languages or stereotypes, inappropriate use of grammar and pronouns or play imitation $^{22}$. However, some individuals with ASD may have specific impairment or other limited comprehensive deficits in gestures or verbal forms of communication that exhibits generalization of language disorders. These findings of our study was consistent with the study of Kleinhans et al $(2016)^{24}$ that language is important for understanding between parent and child, moreover it has been stated that language impairment is one of the main purpose for the behavior issues in ASD children. Another study revealed that scores of non-verbal ability of receptive may vary in communication in presence of language impairment that may leads to behavioral issues, anxiety and depression in the child and parents ${ }^{25}$.

Several studies reported that communicative barriers lead to unbounded relationship between parents and the child. However, it was also observed in our study as majority of parents find it hard to understand their children needs and communication ${ }^{20-24}$. Also, stress is found to be more prevalent in parents with ASD children as compared to those with normal development. Number of studies observed that parental stress increased after diagnosis of autism in their children that eventually leads to communication difficulties, therefore certain interventions are required to focus on strategies that reduces these difficulties. Moreover, lower efficacy, in-efficient role in child's development, culture or community interference has been reported ${ }^{18-22}$. Similarly, our study reported varied perception of their child in understanding that needed to address. Furthermore, some evidence suggested that parent sensitivity not only benefit the child but also reduce parental ignorance towards the diagnosis of the condition ${ }^{25}$.

Evidences from the researchers reported that therapy consisted of parent-child interaction or child-directed may be effective to deal with communicative disorders ${ }^{25}$. These approaches are structured and disciplined-oriented thus effective for the management of disorders although, these programs are implemented limitedly. Also, these approaches are pivotal to reduce parental stress as well as gap between parent-child thereby strengthen their bond through directed interaction and reinforcement that may lead to progress a healthy relationship and understanding. Literature showed that parenting-mediated interventions are essential for parent and child for beneficial health outcome ${ }^{19-22}$. Another study revealed that maternal parenting behaviors are crucial for the child to develop responsiveness there- by decrease the behavior disruption and reduces the maternal stress ${ }^{2-10}$. Therefore, these approaches must be taken in to account for effective implementation.

Despite of the fact, these interventions showed positive gain in language skills, expression and language comprehension thus, decreases the severity of autism characteristics ${ }^{23-24}$. On the contrary, verbal-based interventions are found to be effectively sound to enhance pronunciation, verbal strategies, comprehend expressions through child-centered and pragmatic approaches, including didactic methods, pre-linguistics or milieu communication ${ }^{22}$. Although, these methods uses modeling of communicative behavior based on the child responses and interests. Further these approaches address time delay i.e. waiting for the child to initiate response in natural environment and behavioral corrections ${ }^{20-24}$.

Subsequently, number of approaches is viable to be implemented in ASD rehabilitation, however only few are used to address the ASD characteristics. This is due to limitation of resources or sample constraint that causes barriers to use the tools. Moreover, it is also important to screen for the disorders through standardized and reliable scales/questionnaires for effective evaluation. It is also suggested that parent-child interaction should be incorporated as a part of rehabilitation curriculum to make their understanding clear, as parents participation may influence the child motivation to perform language pragmatics with efficiency. Also, peer relationship should be promoted to prevent child from isolation thereby reducing the disorder stress. Therefore, large scale surveys are recommended to evaluate communicative difficulties through standardized approaches and address the child needs.

\section{CONCLUSION}

It was concluded that varied percentage was observed in communicative domains of the questionnaire that showed high proportion of communication barriers between parents and child. Further studies are needed to analyze the communication between parents and autistic children to address its impact. Thus, parent-child interaction approaches should be used for beneficial outcomes.

\section{REFERENCES}

[1] Lord C, Elsabbagh M, Baird G, Veenstra-Vanderweele J. Autism spectrum disorder. The Lancet. $2018 ; 392(10146): 508-20$.

[2] Abubakar A, Ssewanyana D, Newton CR. A systematic review of research on autism spectrum disorders in Sub-Saharan Africa. Behav. Neurol. 2016.

[3] Postorino V, Fatta LM, Sanges V, Giovagnoli G, De Peppo L, Vicari S, Mazzone L. Intellectual disability in autism spectrum disorder: investigation of prevalence in an Italian sample of 
children and adolescents.

[4] Masi A, DeMayo MM, Glozier N, Guastella AJ. An overview of autism spectrum disorder, heterogeneity and treatment options. Neurosci. Bull. $2017 ; 33(2): 183-93$.

[5] Burzawa Kr. Difficulties In Verbal And Non-Verbal Communication In Autism. Sabiedriba. Integrācija. Izg「itība. $2018 ; 45: 45$.

[6] Varcin KJ, Alvares GA, Uljarević M, Whitehouse AJ. Prenatal maternal stress events and phenotypic outcomes in Autism Spectrum Disorder. Autism Research. $2017 ; 10(11)$ : 1866-77.

[7] Forsyth A, Herrman E, Raslan K, Ortiz S, Lee C, Galhano A. Autism Spectrum Disorder in Children: Behavioral, Sensory and Gastrointestinal Considerations and Assessment of Oral Health. J Dent Oral Biol. 2018; 3 (4). 2018;1138.

[8] Li M, Fallin MD, Riley A, Landa R, Walker SO, Silverstein $M$, Caruso D, Pearson $C$, Kiang $S$, Dahm JL, Hong $X$. The association of maternal obesity and diabetes with autism and other developmental disabilities. Pediatrics. 2016 ;137(2):e20152206.

[9] Gernsbacher MA, Morson EM, Grace EJ. Language and speech in autism. Annu. Rev. Linguist. $2016 ; 2: 413-25$.

[10] Stevenson RA, Baum SH, Segers M, Ferber S, Barense MD, Wallace MT. Multisensory speech perception in autism spectrum disorder: From phoneme to whole-word perception. Autism Research. 2017;10(7):1280-90.

[11] Frantz R, Hansen SG, Machalicek W. Interventions to promote well-being in parents of children with autism: A systematic review. J Autism Dev Disord. $2018 ; 5(1): 58-77$.

[12] Da Paz NS, Wallander JL, Tiemensma J. Effects of written disclosure on psychophysiological stress among parents of children with autism: A randomized controlled pilot study. Research in Autism Spectrum Disorders. 2018 ;53:7-17.

[13] Sutherland R, Trembath D, Hodge MA, Rose V, Roberts J. Telehealth and autism: Are telehealth language assessments reliable and feasible for children with autism?. Int J Lang Comm Dis. 2019;54(2):281-91.

[14] Bolton J. M. Cl. Sc (SLP) Candidate University of Western Ontario: School of Communication Sciences and Disorders the following appraisal examined the evidence for increased verbal output in non-verbal children with Autism Spectrum Disorder (ASD) after engaging in the picture exchange communication system.

[15] Bishop DV. Why is it so hard to reach agreement on terminology? The case of develop- mental language disorder (DLD). Int J Lang Comm Dis. 2017;52(6):671-80.

[16] Deliens G, Papastamou F, Ruytenbeek N, Geelhand $P$, Kissine M. Selective pragmatic impairment in autism spectrum disorder: Indirect requests versus irony. J Autism Dev Disord. 2018;48(9):2938-52.

[17] Di Renzo M, Bianchi di Castelbianco F, Vanadia E, Petrillo M, Racinaro L, Rea M. TULIP protocol (TCE, UOI, Leiter-R as indicators of predictivity) for the assessment of the developmental potential in children with autism spectrum disorders. Autism Open Access. 2016;6(4): 188-94.

[18] Stokes MA, Kornienko L, Scheeren AM, Koot $H M$, Begeer S. A comparison of children and adolescent's self-report and parental report of the PedsQL among those with and without autism spectrum disorder. Qual. Life Res.2017; 26(3):611-24

[19] Salcuni S, Mazzeschi C, Capella C. The role of play in child assessment and intervention. Frontiers in psychology. $2017 ; 8: 1098$.

[20] Wolff, L. and Benge, J., 2019. Everyday Language Difficulties in Parkinson's Disease: Caregiver Description and Relationship with Cognition, Activities of Daily Living, and Motor Disability. Am J Speech Lang Pathol. 28(1), pp.165-173.

[21] Invernizzi PL, Crotti M, Bosio A, Cavaggioni L, Alberti G, Scurati R. Multi-teaching styles approach and active reflection: Effectiveness in improving fitness level, motor competence, enjoyment, amount of physical activity, and effects on the perception of physical education lessons in primary school children. Sustainability. $2019 ; 11$ (2):405.

[22] Balestro J, Fernandes F. Questionnaire about communicative difficulties perceived by parents of children of the autism spectrum. Rev soc bras fonoaudiol. 2012;3.

Velasquez F, Qin XA, Reilly MA, Neuhaus E,

[23] Estes A, Aylward E, Kleinhans NM. Neural correlates of emotional inhibitory control in autism spectrum disorders. Res Dev Disabil. $2017 ; 64: 64-77$.

[24] Kleinhans NM, Richards T, Greenson J, Dawson $G$, Aylward E. Altered dynamics of the fMRI response to faces in individuals with autism. J Autism Dev Disord . 2016 ;46(1):232-41.

[25] Watkins L, Kuhn M, Ledbetter-Cho K, Gevarter C, O'Reilly M. Evidence-based social communication interventions for children with autism spectrum disorder. Indian J. Pediatr. 2017 84(1):68-75. 